\title{
Maximum Power Point Tracking of Thermoelectric Generation Systems Under Nonuniform Temperature Distribution: A State-of-the-Art Evaluation
}

\author{
Ruining Shao ${ }^{1}$, Bo Yang ${ }^{1}$, Nuo Chen ${ }^{2}$ and Yiming Han ${ }^{1 *}$ \\ ${ }^{1}$ Faculty of Electric Power Engineering, Kunming University of Science and Technology, Kunming, China, ${ }^{2}$ China Southern Power \\ Grid EHV Transmission Company, Kunming, China
}

Keywords: solar energy, thermoelectric generation, non-uniform temperature distribution, maximum power point tracking, optimization

\section{INTRODUCTION}

OPEN ACCESS

Edited by:

Bin Zhou,

Hunan University, China

Reviewed by:

Xuehan Zhang,

Korea University, South Korea

Jian Chen,

Yancheng Institute of Technology,

China

*Correspondence:

Yiming Han

373482753@qq.com

Specialty section: This article was submitted to Process and Energy Systems

Engineering,

a section of the journal Frontiers in Energy Research

Received: 18 January 2022 Accepted: 27 January 2022 Published: 04 March 2022

Citation:

Shao $R$, Yang B, Chen $N$ and Han $Y$ (2022) Maximum Power Point Tracking

of Thermoelectric Generation Systems

Under Nonuniform Temperature

Distribution: A State-of-the-

Art Evaluation

Front. Energy Res. 10:857261.

doi: 10.3389/fenrg.2022.857261
Over the past few years, due to the increasing demand for energy and the decreasing reserves of fossil energy, renewable energy has attracted more attention and gradually replaced most of the fossil fuels, among which solar energy is one of the most promising one (Zhang et al., 2015; Zhang et al., 2016; Yao et al., 2019). Recently, thermoelectric generation (TEG) is an important application technology of solar power generation fields (Iqbal et al., 2021; Zhao et al., 2021), which usually serves as a thermoelectric waste heat energy recovery system in hybrid power generation system (Chen et al., 2021). However, due to the low conversion efficiency, expensive material cost, temperature mismatch, and variation of internal resistance of TEG system, improving technologies and more efficient TEG material are exploited to accelerate the industrialization of TEG system (Liu et al., 2016). In terms of the above technologies, maximum power point tracking (MPPT) is a necessary and crucial technique to extract the maximum power during the operating of the TEG system. However, MPPT for the TEG system will face a lot of challenges. For the TEG system, nonuniform temperature distribution (NUTD) condition limits the available power. Under this nonuniform circumstance, the output power-voltage $(P-V)$ characteristics will exhibit several peaks, which makes MPPT more difficult (Yang et al., 2020a). In this context, many kinds of MPPT algorithms emerged in recent years. This paper gives some viewpoints of the TEG systems and existing MPPT algorithms for the TEG systems, as well as some suggestions for future research.

\section{MODEL OF THE THERMOELECTRIC GENERATION SYSTEM}

The basic structure of the TEG system is illustrated in Figure 1. In a closed circuit of two different conducting materials, when the two contacts are at different temperatures, the potential generated in the circuit converts heat energy into electricity, and this phenomenon is called Seebeck effect (Zhu et al., 2021). In the TEG system, p-type and n-type semiconductors are connected with cold side conductor material and hot side conductor material to increase system voltage level. The main factors influencing conversion efficiency of the TEG system are Seebeck coefficient $\alpha$, electrical conductivity $\sigma$, and thermal conductivity $k$. The figure of merit $Z$ is an evaluation criteria of efficiency of the TEG material, which is shown as follows: 


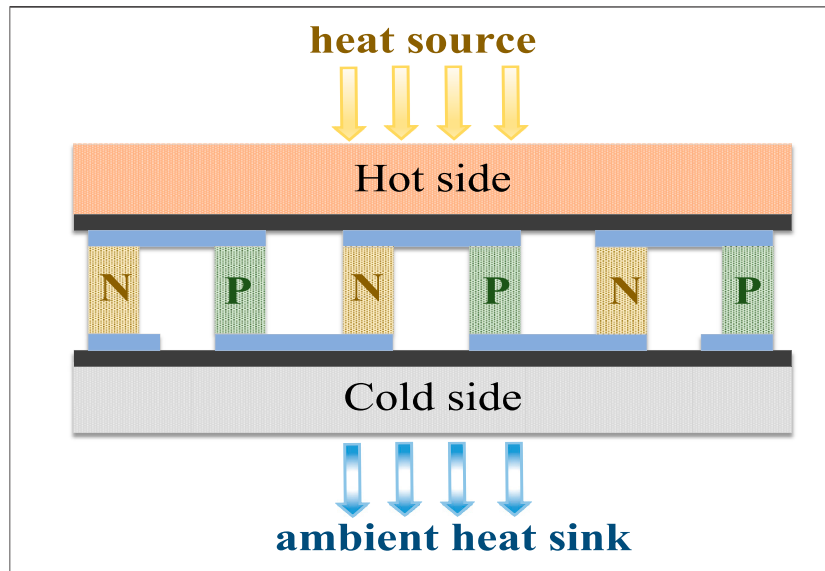

FIGURE 1 | Basic structure of the thermoelectric generation (TEG) system.

$$
Z=\frac{\alpha^{2} \sigma}{k}
$$

It can be concluded that a thermoelectric material with higher electricity conductivity and higher Seebeck coefficient are key factors in choosing high-performance TEG materials, and materials with high thermal conductivity can be used as a cooling device in the TEG system. The optimization of a cooling device in TEG is the main market of current commercial TEG (Manikandan and Kaushik, 2015). A study (Sato and Yamada, 2019) explored the different photovoltaic module cooling methods, which can provide a reference for cooling devices of the TEG system. Besides, utilization of waste heat can be improved by increasing heat flux through TEG; one effective way is to install heat pipes in TEG modules (Wen et al., 2021). Selecting a proper number of thermocouples and designing a reasonable structure of DC-DC converters are also crucial to make maximum use of the generated power by the TEG system. Apart from methods aforementioned, an optimized system design is another useful method to enhance the efficiency of the TEG system. The interconnection of the TEG modules in large-scale systems is limited by actual installation conditions, resulting in parameter mismatch of the TEG modules and mismatch loss. So it is necessary to further study the topology of large-scale gridconnected TEG systems. A study (Al-Habahbeh et al., 2016) explored the geometric design of a large TEG system and optimization of the key parameters of the system, which deserves further studying. Therefore, there is a need to create standard mathematical models for researchers to conduct effective research. It is more reasonable to consider factors that influence temperature in a practical scenario, which will affect the accuracy of mathematical models (Dasu et al., 2021; Sakthivel and Sathya, 2021).

\section{MAXIMUM POWER POINT TRACKING FOR THERMOELECTRIC GENERATION SYSTEM}

\section{Non-Uniform Temperature Distribution}

Mismatch of the TEG system are usually caused by NUTD, aging, and faults on TEG modules, which influences the efficiency and service life of the TEG system. In practice, TEGs usually operate under dynamical environments with time-varying temperature differences called NUTD. Under this circumstance, there will emerge multiple MPPs, which hinder the tracking of GMPP. Literature about MPPT for the TEG system usually use step change in temperature and random temperature as NUTD condition in case studies (Yang et al., 2019a; Yang et al., 2020b; Majad et al., 2021; Yang et al., 2021). These can be summed up as the evaluation criteria of case studies in the MPPT for the TEG system. A study (A. et al., 2021) collected field temperature and irradiance data between 10:00 a.m. and 1:00 p.m. to evaluate the effects of real environment parameters, such as angle of installation or irradiation on temperature of the TEG system, providing the latest reference data available for researchers. Temperature level, duration, heat loss during heat conduction, and other rapid variations in NUTD should be considered in practical engineering.

Therefore, by choosing the proper models of time-varying NUTD, applicable topology of the TEG system, MPPT algorithms, and other advanced mismatch mitigating techniques can relieve the adverse impact of NUTD.

\section{Maximum Power Point Tracking of Thermoelectric Generation System}

MPPT techniques of the TEG system can be classified into classical ones and intelligent ones. Traditional MPPT algorithms are perturb and observe $(\mathrm{P} \& \mathrm{O})$ algorithm, hill climbing (HC) technique, and incremental conductance (INC) technique (Eakburanawat and Boonyaroonate, 2006; Rae-Young Kim et al., 2009; Shang et al., 2020). Almost all MPPT algorithms can extract the global maximum power point (GMPP) under uniform temperature condition (Yang et al., 2019b), but the above classic techniques are easily trapped into the local maximum power point (LMPP) and might have steady-state oscillations. There are also some improved algorithms of traditional methods, which adopted variable step size instead of fixed steps, so as to enhance tracking precision and balance between steady-state oscillations and response speed to some certain extent, but there are still some issues of low tracking speed and getting easily trapped in local optimum (Shiriaev et al., 2019). A study (Kanagaraj et al., 2020) proposed a variable fractional order fuzzy logic control MPPT algorithm, which adjusted fractional factor $\alpha$ to shorten the tracking time. Another study (Liu et al., 2016) combined the P\&O method and open circuit voltage $(\mathrm{OCV})$ method to realize a faster and simpler tracking, but the aforementioned methods are based on trial-and-error principle, in which the operating point usually oscillates around MPP in a steady state. A study (Bijukumar et al., 2018) used two measurable operating points to calculate optimal duty ratio under MPP, which has high precision and have no steady-state oscillation around MPP. Recently, there are many advanced MPPT algorithms that emerged, such as metaheuristic algorithms or mathematics-based algorithms. Metaheuristic algorithms are increasingly used in recent studies due to their high efficiency, simple mechanism, and not being easily trapped in LMPP. Among the above techniques, swarm intelligence (SI)- 
based MPPT techniques outperform other methods due to it not requiring an exact mathematical model and not easily converging to local optimum. Up to now, adaptive compass search (ACS) (Yang et al., 2019a), equilibrium optimization (EQO) (Majad et al., 2021), fast atom search optimization (FASO) (Yang et al., 2020b), interacted collective intelligence (ICI) (Yang et al., 2021), sine cosine algorithm (SCA) (Rezk et al., 2021), and many other intelligent algorithms have been studied. Basically, they carried out four case studies, which are startup test, step change in temperature, random temperature variation, and sensitivity analysis, respectively. These can be a standard for case studies in relevant fields. A study (Rezk et al., 2021) compared the best, the worst, the average, median, variance, and standard deviation of MPPT results to evaluate the performance of the particle swarm algorithm (PSO), whale optimization algorithm (WOA), and SCA, which can be used as references in testing new MPPT algorithms for researchers. Moreover, metaheuristic algorithm-based MPPT techniques are usually of high randomness, and the execution time increases as the scale of TEG increases. So, there is a need to explore more stable metaheuristic algorithms with general applicability, and there should be more hardware experimental setup to verify the validity and accuracy of the proposed methods.

Up to now, literature regarding the assessment of MPPT for a large TEG plant is limited to a few cases. A study (Molina et al., 2010) discussed two hardware topologies of MPPT, which are, respectively, one-stage topology and two-stage topology, then proposed a two-stage configuration for the distributed TEG system. This flexibility in the design of the MPPT topology is worth advocating. In addition, it is worth considering how many MPP trackers and converters should be used in MPPT studies, which is a practical issue for system design. Anyway, the design of the MPPT system should be combined with actual installation condition in engineering projects. At present, the topology of the TEG system in MPPT studies does not have a uniform standard, which needs to be further established. For a large-scale TEG system, the system designer should decide to use how many MPP trackers or converters under different installation conditions. Furthermore, the ambient irradiance, number of thermocouples, and other environmental inputs in simulation should use all-purpose nominal specifications to get the exact result. How to set these parameters properly is a problem that researchers need to consider (Chen et al., 2018; Zhou et al., 2020; Huang et al., 2021; Xiong et al., 2021).

\section{HYBRID PV-THERMOELECTRIC GENERATION SYSTEM}

In PV systems, the waste heat and rise in temperature result from solar irradiation greatly reducing the energy conversion efficiency or even damaging the PV panel. The PV-TEG system with heat sinks can recycle the waste heat from a PV panel, which is a promising and worth investigating improved technique. So far, there has been little research on MPPT for the PV-TEG systems. A study (Adeel et al., 2020) proposed the arithmetic optimization algorithm (AOA) for MPPT, which applied nonuniform irradiance and nonuniform temperature distribution as a study case to evaluate the proposed method. A study (Kanagaraj, 2021) used step change in solar irradiation and a constant temperature difference to evaluate FOFLC, $\mathrm{P} \& \mathrm{O}$, and FLC MPPT techniques of the PV-TEG system. These are open to question because temperatures of TEG modules mostly depend on temperatures of PV modules. In other words, it is unrealistic to design irradiation and temperature difference separately. It is more appropriate to combine both of the aforementioned to meet the demands of the study. Literature (Sark, 2011) determined the temperature of TEG modules according to ambient temperature and irradiation, which is given by Eq. 2. Studies (Verma et al., 2016; Babu and Ponnambalam, 2018) considered the influence of wind speed on temperature, which is computed as Eqs. 3 and 4:

$$
\begin{gathered}
T_{\mathrm{TEG} 1}=\frac{1}{2}\left(T_{\mathrm{M}}+T_{\mathrm{A}}\right)=T_{\mathrm{A}}+\frac{1}{2} c G \\
T_{\mathrm{TEG} 2}=0.943 T_{A}+0.028 G \cdot 1000-1.521 w_{s}+4.3 \\
T_{\mathrm{TEG} 3}=0.943 T_{A}+0.0195 G \cdot 1000-1.528 w_{s}+0.3529
\end{gathered}
$$

where $T_{\mathrm{TEG} 1}, T_{\mathrm{TEG} 2}$, and $T_{\mathrm{TEG} 3}$ are the average temperatures of the TEG module in the above literature, respectively; $T_{M}$ is the temperature of the PV module; $T_{\mathrm{A}}$ is the ambient temperature; $c$ is a coefficient determined by installation conditions; and $G$ is the irradiance.

In addition, it is worth investigating for researchers to study more efficient and systematic metaheuristic algorithms, thus, realizing efficient MPPT for the PV-TEG system (Liu et al., 2020; Wang et al., 2021; Zhang et al., 2021).

\section{DISCUSSION AND CONCLUSION}

MPPT algorithms are the most frequently used techniques to obtain the maximum power of the TEG systems, but there is still room for improvement. Researchers can promote research priorities for MPPT of the TEG system to fill up the gaps in the previous studies. Recommendations and limitations of this technique are as follows:

(a) NUTD in a real scenario can be further simulated, such as the variation in temperature of the TEG system from sunrise to sunset in 1 day. In addition, standard study cases of NUTD for researchers to simulate in MPPT studies can be further established.

(b) For the TEG system, researchers should give more consideration to the mathematical model of system, in which wind speed, installation condition, and other practical factors can be considered.

(c) Existing MPPT algorithms are only available for small-scale systems. Most of the literature only conducts simulation under uneven distribution of temperature. Hence, there is a need to consider other factors leading to mismatch and study how many converters or MPP trackers should be used in large-scale system. 
(d) MPPT techniques for the hybrid PV-TEG system have a large potential. Researchers can further study the MPPT techniques of the hybrid system, which are underexploited in the related fields.

Future studies will further explore the following aspects:

(i) MPPT techniques for actual large-scale TEG system will be further explored to meet the demand of practical engineering.

\section{REFERENCES}

Adeel, F. M., Majad, M., Kamal, Z., Javed, M. Y., Zafar, M. H., and Khan, N. M. (2020). High-efficiency Hybrid PV-TEG System with Intelligent Control to Harvest Maximum Energy under Various Non-static Operating Conditions. J. Clean. Prod. 320, 128643. doi:10.1016/j.jclepro.2021.128643

Al-Habahbeh, O. M., Mohammad, A., Al-Khalidi, A., Khanfer, M., and Obeid, M. (2016). Design Optimization of a Large-Scale Thermoelectric Generator. J. King Saud Univ. - Eng. Sci. 30, 177. doi:10.1016/j.jksues.2016.01.007

A., R., S, S., Hassan, W. Z. W., Azis, N., Ya'acob, M. E., Elianddy, E., et al. (2021). Performance Analysis of Thermoelectric Generator Implemented on Nonuniform Heat Distribution of Photovoltaic Module. Energ. Rep. 7, 2379-2387. doi:10.1016/j.egyr.2021.04.029

Babu, C., and Ponnambalam, P. (2018). The Theoretical Performance Evaluation of Hybrid PV-TEG System. Energ. Convers. Manage. 173, 450-460. doi:10. 1016/j.enconman.2018.07.104

Bijukumar, B., Raam, A. G. K., Ganesan, S. I., and Nagamani, C. (2018). A Linear Extrapolation-Based MPPT Algorithm for Thermoelectric Generators under Dynamically Varying Temperature Conditions. IEEE Trans. Energ. Convers. 33, 1641-1649. doi:10.1109/tec.2018.2830796

Chen, J., Yang, R., Xu, D., Zhou, B., and Jin, Y. (2021). Maximum Production point Tracking Method for a Solar-Boosted Biogas Energy Generation System. Prot. Control. Mod. Power Syst. 6 (42), 1-13. doi:10.1186/s41601-021-00220-Z

Chen, J., Yao, W., Zhang, C., Ren, Y., and Jiang, L. (2018). Design of Robust MPPT Controller for Grid-Connected PMSG-Based Wind Turbine via Perturbation Observation Based Nonlinear Adaptive Control. Renew. Energ. 101, 34-51.

Dasu, B., Mangipudi, S., and Rayapudi, S. (2021). Small Signal Stability Enhancement of a Large Scale Power System Using a Bio-Inspired Whale Optimization Algorithm. Prot. Control. Mod. Power Syst. 6 (16), 1-17. doi:10. 1186/s41601-021-00215-w

Eakburanawat, J., and Boonyaroonate, I. (2006). Development of a Thermoelectric Battery-Charger with Microcontroller-Based Maximum Power point Tracking Technique. Appl. Energ. 83 (7), 687-704. doi:10.1016/j.apenergy.2005.06.004

Huang, S., Wu, Q., Liao, W., Wu, G., Li, X., and Wei, J. (2021). Adaptive DroopBased Hierarchical Optimal Voltage Control Scheme for Vsc-Hvdc Connected Offshore Wind Farm. IEEE Trans. Ind. Inf. 17 (12), 8165-8176. doi:10.1109/tii. 2021.3065375

Iqbal, B., Nasir, A., and Murtaza, A. F. (2021). Stochastic Maximum Power point Tracking of Photovoltaic Energy System under Partial Shading Conditions. Prot. Control. Mod. Power Syst. 6 (30), 1-13. doi:10.1186/s41601-021-00208-9

Kanagaraj, N. (2021). Photovoltaic and Thermoelectric Generator Combined Hybrid Energy System with an Enhanced Maximum Power point Tracking Technique for Higher Energy Conversion Efficiency. Sustainability 13 (6), 3144. doi:10.3390/su13063144

Kanagaraj, N., Rezk, H., and Gomaa, M. R. (2020). A Variable Fractional Order Fuzzy Logic Control Based MPPT Technique for Improving Energy Conversion Efficiency of Thermoelectric Power Generator. Energies 13 (17), 1-18. doi:10. 3390/en13174531

Liu, J., Yao, W., Wen, J., Fang, J., Jiang, L., He, H., et al. (2020). Impact of Power Grid Strength and PLL Parameters on Stability of Grid-Connected DFIG Wind Farm. IEEE Trans. Sustain. Energ. 11 (1), 545-557. doi:10.1109/tste.2019. 2897596 (ii) Efficient and more stable metaheuristic algorithm-based MPPT method for the TEG system will be designed to fill the gap of related fields.

\section{AUTHOR CONTRIBUTIONS}

RS: writing the original draft and editing. BY: conceptualization. NC: visualization and contributed to the discussion of the topic. YH: visualization and contributed to the discussion of the topic.

Liu, Y.-H., Chiu, Y.-H., Huang, J.-W., and Wang, S.-C. (2016). A Novel Maximum Power point Tracker for Thermoelectric Generation System. Renew. Energ. 97, 306-318. doi:10.1016/j.renene.2016.05.001

Majad, M., Adeel, F. M., Shihui, D., Zhu, J., Yin, B., and Ling, Q. (2021). Maximum Energy Harvesting of Centralized Thermoelectric Power Generation Systems with Non-uniform Temperature Distribution Based on Novel Equilibrium Optimizer. Energ. Convers. Manage. 246, 114694. doi:10.1016/j.enconman. 2021.114694

Manikandan, S., and Kaushik, S. C. (2015). Thermodynamic Studies and Maximum Power point Tracking in Thermoelectric GeneratorThermoelectric Cooler Combined System. Cryogenics 67, 52-62. doi:10. 1016/j.cryogenics.2015.01.008

Molina, M. G., Juanicó, L. E., Rinalde, G. F., Taglialavore, E., and Gortari, S. (2010). Design of Improved Controller for Thermoelectric Generator Used in Distributed Generation. Int. J. Hydrogen Energ. 35 (11), 5968-5973. doi:10. 1016/j.ijhydene.2009.12.098

Rae-Young Kim, R. Y., Jih-Sheng Lai, J. S., York, B., and Koran, A. (2009). Analysis and Design of Maximum Power point Tracking Scheme for Thermoelectric Battery Energy Storage System. IEEE Trans. Ind. Electron. 56 (9), 3709-3716. doi:10.1109/tie.2009.2025717

Rezk, H., Alhato, M. M., Al-Dhaifallah, M., and Bouallègue, S. (2021). A Sine Cosine Algorithm-Based Fractional MPPT for Thermoelectric Generation System. Sustainability 13, 11650. doi:10.3390/su132111650

Sakthivel, V. P., and Sathya, P. D. (2021). Single and Multi-Area Multi-Fuel Economic Dispatch Using a Fuzzified Squirrel Search Algorithm. Prot. Control. Mod. Power Syst. 6 (11), 1-13. doi:10.1186/s41601-021-00188-w

Sark, W. G. J. H. M. V. (2011). Feasibility of Photovoltaic - Thermoelectric Hybrid Modules. Appl. Energ. 88 (8), 2785-2790. doi:10.1016/j.apenergy.2011.02.008

Sato, D., and Yamada, N. (2019). Review of Photovoltaic Module Cooling Methods and Performance Evaluation of the Radiative Cooling Method. Renew. Sustain. Energ. Rev. 104, 151-166. doi:10.1016/j.rser.2018.12.051

Shang, L., Guo, H., and Zhu, W. (2020). An Improved MPPT Control Strategy Based on Incremental Conductance Algorithm. Prot. Control. Mod. Power Syst. 5 (14), 1-8. doi:10.1186/s41601-020-00161-z

Shiriaev, P., Shishov, K., and Osipkov, A. (2019). Electrical Network of the Automotive Multi-Sectional Thermoelectric Generator with MPPT Based Device Usage. Mater. Today Proc. 8, 642-651. doi:10.1016/j.matpr.2019.02.064

Verma, V., Kane, A., and Singh, B. (2016). Complementary Performance Enhancement of PV Energy System through Thermoelectric Generation. Renew. Sustain. Energ. Rev. 58, 1017-1026. doi:10.1016/j.rser.2015.12.212

Wang, P., Song, J., Liang, F., Shi, F., Kong, X., Xie, G., et al. (2021). Equivalent Model of Multi-type Distributed Generators under Faults with Fast-Iterative Calculation Method Based on Improved PSO Algorithm. Prot. Control. Mod. Power Syst. 6 (29), 1-12. doi:10.1186/s41601-021-00207-w

Wen, X., Ji, J., Song, Z., Li, Z., Xie, H., and Wang, J. (2021). Comparison Analysis of Two Different Concentrated photovoltaic/thermal-TEG Hybrid Systems. Energ. Convers. Manage. 234, 113940. doi:10.1016/j.enconman.2021.113940

Xiong, Y., Yao, W., Wen, J., Lin, S., Ai, X., Fang, J., et al. (2021). Two-level Combined Control Scheme of VSC-MTDC Integrated Offshore Wind Farms for Onshore System Frequency Support. IEEE Trans. Power Syst. 36 (1), 781-792. doi:10.1109/tpwrs.2020.2998579

Yang, B., Wang, J., Zhang, X., Zhang, M., Shu, H., Li, S., et al. (2019). MPPT Design of Centralized Thermoelectric Generation System Using Adaptive Compass 
Search under Non-uniform Temperature Distribution Condition. Energ. Convers. Manage. 199, 111991. doi:10.1016/j.enconman.2019.111991

Yang, B., Zhang, M., Wang, J., Zeng, K., Zhang, Z., Shu, H., et al. (2021). Interacted Collective Intelligence Based Energy Harvesting of Centralized Thermoelectric Generation Systems under Non-uniform Temperature Gradient. Sustainable Energ. Tech. Assessments 48, 101600. doi:10.1016/j.seta.2021.101600

Yang, B., Zhang, M., Zhang, X., Wang, J., Shu, H., Li, S., et al. (2020). Fast Atom Search Optimization Based MPPT Design of Centralized Thermoelectric Generation System under Heterogeneous Temperature Difference. J. Clean. Prod. 248, 119301. doi:10.1016/j.jclepro.2019.119301

Yang, B., Zhong, L., Zhang, X., Shu, H., Yu, T., Li, H., et al. (2019). Novel BioInspired Memetic Salp Swarm Algorithm and Application to MPPT for PV Systems Considering Partial Shading Condition. J. Clean. Prod. 215, 1203-1222. doi:10.1016/j.jclepro.2019.01.150

Yang, B., Zhu, T., Wang, J., Shu, H., Yu, T., Zhang, X., et al. (2020). Comprehensive Overview of Maximum Power point Tracking Algorithms of PV Systems under Partial Shading Condition. J. Clean. Prod. 268, 121983. doi:10.1016/j.jclepro. 2020.121983

Yao, Q., Liu, J., and Hu, Y. (2019). Optimized Active Power Dispatching Strategy Considering Fatigue Load of Wind Turbines during De-loading Operation. IEEE Access 7, 17439-17449. doi:10.1109/access.2019.2893957

Zhang, K., Zhou, B., Or, S. W., Li, C., Chung, C. Y., and Voropai, N. I. (2021). Optimal Coordinated Control of Multi-Renewable-To-Hydrogen Production System for Hydrogen Fueling Stations. IEEE Trans. Ind. Applicat. 2021, 1. doi:10.1109/TIA.2021.3093841

Zhang, X., Yu, T., Yang, B., and Li, L. (2016). Virtual Generation Tribe Based Robust Collaborative Consensus Algorithm for Dynamic Generation Command Dispatch Optimization of Smart Grid. Energy 101, 34-51. doi:10. 1016/j.energy.2016.02.009

Zhang, X., Yu, T., Yang, B., Zheng, L., and Huang, L. (2015). Approximate Ideal Multi-Objective Solution $\mathrm{Q}(\lambda)$ Learning for Optimal Carbon-Energy Combined-Flow in Multi-Energy Power Systems. Energ. Convers. Manage. 106, 543-556. doi:10.1016/j.enconman.2015.09.049
Zhao, Y., An, A., Xu, Y., Wang, Q., and Wang, M. (2021). Model Predictive Control of Grid-Connected PV Power Generation System Considering Optimal MPPT Control of PV Modules. Prot. Control. Mod. Power Syst. 6 (32), 1-12. doi:10. 1186/s41601-021-00210-1

Zhou, B., Fang, J., Ai, X., Yang, C., Yao, W., and Wen, J. (2020). Dynamic Var reserve-constrained Coordinated Scheduling of LCC-HVDC Receiving-End System Considering Contingencies and Wind Uncertainties. IEEE Trans. Sustain. Energ. 12 (1), 469-481.

Zhu, M., Xu, C., Dong, S., Tang, K., and Gu, C. (2021). An Integrated Multi-Energy Flow Calculation Method for Electricity-Gas-thermal Integrated Energy Systems. Prot. Control. Mod. Power Syst. 6 (5), 1-12. doi:10.1186/s41601021-00182-2

Conflict of Interest: NC is employed by the China Southern Power Grid EHV Transmission Company.

The remaining authors declare that the research was conducted in the absence of any commercial or financial relationships that could be construed as a potential conflict of interest.

Publisher's Note: All claims expressed in this article are solely those of the authors and do not necessarily represent those of their affiliated organizations, or those of the publisher, the editors, and the reviewers. Any product that may be evaluated in this article, or claim that may be made by its manufacturer, is not guaranteed or endorsed by the publisher.

Copyright () 2022 Shao, Yang, Chen and Han. This is an open-access article distributed under the terms of the Creative Commons Attribution License (CC $B Y$ ). The use, distribution or reproduction in other forums is permitted, provided the original author(s) and the copyright owner(s) are credited and that the original publication in this journal is cited, in accordance with accepted academic practice. No use, distribution or reproduction is permitted which does not comply with these terms. 